\title{
BMJ Open Faecal calprotectin to detect inflammatory bowel disease: a systematic review and exploratory meta- analysis of test accuracy
}

\author{
Karoline Freeman, ${ }^{\oplus 1}$ Brian H Willis, ${ }^{2}$ Hannah Fraser, ${ }^{1}$ Sian Taylor-Phillips, ${ }^{\odot 1}$ \\ Aileen Clarke ${ }^{1}$
}

To cite: Freeman $\mathrm{K}$, Willis $\mathrm{BH}$, Fraser $\mathrm{H}$, et al. Faecal calprotectin to detect inflammatory bowel disease: a systematic review and exploratory meta-analysis of test accuracy. BMJ Open 2019;9:e027428. doi:10.1136/ bmjopen-2018-027428

- Prepublication history and additional material for this paper are available online. To view these files, please visit the journal online (http://dx.doi. org/10.1136/bmjopen-2018027428).

Received 22 October 2018 Revised 9 January 2019 Accepted 28 January 2019

Check for updates

(c) Author(s) (or their employer(s)) 2019. Re-use permitted under CC BY. Published by BMJ.

${ }^{1}$ Warwick Medical School, University of Warwick, Coventry, UK

${ }^{2}$ Institute of Applied Health Research, University of Birmingham, Birmingham, UK

Correspondence to

Karoline Freeman;

K.Freeman@warwick.ac.uk

\section{ABSTRACT}

Objective Test accuracy of faecal calprotectin $(\mathrm{FC})$ testing in primary care is inconclusive. We aimed to assess the test accuracy of FC testing in primary care and compare it to secondary care estimates for the detection of inflammatory bowel disease (IBD).

Methods Systematic review and meta-analysis of test accuracy using a bivariate random effects model. We searched MEDLINE, EMBASE, Cochrane Library and Web of Science until 31 May 2017 and included studies from auto alerts up until 31 January 2018. Eligible studies measured FC levels in stool samples to detect IBD in adult patients with chronic (at least 6-8 weeks) abdominal symptoms in primary or secondary care. Risk of bias and applicability were assessed using the Quality Assessment of Diagnostic Accuracy Studies-2 criteria. We followed the protocol registered as PROSPERO CRD 42012003287.

Results 38 out of 2168 studies were eligible including five from primary care. Comparison of test accuracy by setting was precluded by extensive heterogeneity. Overall, summary estimates of sensitivity and specificity were not recorded. At a threshold of $50 \mu \mathrm{g} / \mathrm{g}$, sensitivity from separate meta-analysis of four assay types ranged from 0.85 (95\% Cl 0.75 to 0.92 ) to 0.94 (95\% Cl 0.75 to 0.90$)$ and specificity from $0.67(95 \% \mathrm{Cl} 0.56$ to 0.76$)$ to 0.88 (95\% $\mathrm{Cl} 0.77$ to 0.94$)$. Across three different definitions of disease, sensitivity ranged from $0.80(95 \% \mathrm{Cl} 0.76$ to $0.84)$ to $0.97(95 \% \mathrm{Cl} 0.91$ to 0.99$)$ and specificity from 0.67 (95\% Cl 0.58 to 0.75$)$ to 0.76 (95\% Cl 0.66 to 0.84$)$. Sensitivity appears to be lower in primary care and is further reduced at a revised threshold of $100 \mu \mathrm{g} / \mathrm{g}$. Conclusions Conclusive estimates of sensitivity and specificity of FC testing in primary care for the detection of IBD are still missing. There is insufficient evidence in the published literature to support the decision to introduce FC testing in primary care. Studies evaluating FC testing in an appropriate primary care setting are needed.

\section{INTRODUCTION}

Inflammatory bowel disease (IBD) is an organic disease caused by inflammation of the intestine. The disease is severe and progressive, ${ }^{12}$ and over $50 \%$ of people require surgery within 10 years of diagnosis. ${ }^{3}$ Timely referral,
Strengths and limitations of this study

- This review used an innovative approach involving random combinations of test accuracy data from included studies in 25000 meta-analyses to display the breadth of heterogeneous evidence from included studies.

- Exploratory meta-analyses investigated different test assays, clinical questions and positivity thresholds of faecal calprotectin levels.

- No overall summary estimates of sensitivity and specificity were produced due to heterogeneity.

- Comparison of test accuracy by setting was precluded by extensive heterogeneity in the small number of studies in primary care.

- The categorisation into different clinical questions was subjective because the disease categories are ill defined and studies' definitions of conditions and groups of conditions varied.

which requires filtering of patients with a high probability of IBD from a broader group of patients with mainly irritable bowel syndrome (IBS), is vital. As many as 3.1 million people in the $\mathrm{USA}^{4}$ and 2.5-3 million people in Europe ${ }^{5}$ suffer from IBD with close to 300000 patients in the UK. ${ }^{6}$ An estimated 256000 new cases of IBD are diagnosed annually throughout Europe. ${ }^{5}$ Northern America and Northern Europe, including the UK, have the highest incidence rates. ${ }^{7}$

General practitioners refer between $10 \%$ and $20 \%$ of patients presenting with abdominal symptoms if no specific test is available. ${ }^{89}$ However, only about $25 \%$ of referred patients have organic disease, of which one-third have IBD. ${ }^{10}{ }^{11}$ Faecal calprotectin (FC), a regulator of the inflammatory response, could potentially aid more selective referral. Patients with high levels of FC have an increased probability of IBD. FC is a small calcium-binding protein. It is released into the intestinal lumen from activated neutrophils accumulating at the 
site of inflammation, and levels of FC are correlated with the level of inflammation. ${ }^{12}$ The protein is stable at room temperature and resistant to digestion, making it a relatively easy candidate for a stool test for inflammation of the intestinal tract.

The FC test is approved by the Food and Drug Administration and recommended by gastroenterological societies across the globe for its usefulness in the diagnosis of IBD. ${ }^{13-16}$ However, there is no clear guidance on which settings it is considered appropriate. FC testing is not supported by Medicare in the USA and Australia, but is approved for the differential diagnosis of IBD and IBS in adult patients in the UK by the National Institute for Health and Care Excellence (NICE) when referral to secondary care is being considered and cancer is not suspected (DG11). ${ }^{17}$ At the time of the recommendations, only test accuracy studies undertaken in secondary care were available to inform the decision. ${ }^{18}$ Our knowledge on how the test performs in primary care is limited and a systematic approach to assessing the applicability of the available evidence specifically for this setting is timely. We aimed to assess the test accuracy of FC testing to detect IBD in adult patients with chronic abdominal symptoms in a primary care pathway (figure 1). In addition, we aimed to explore the differences in FC test performance between primary and secondary care.

\section{METHODS}

\section{Review methods}

We followed the protocol by Waugh et al ${ }^{18}$ registered as PROSPERO CRD 42012003287. We considered all studies

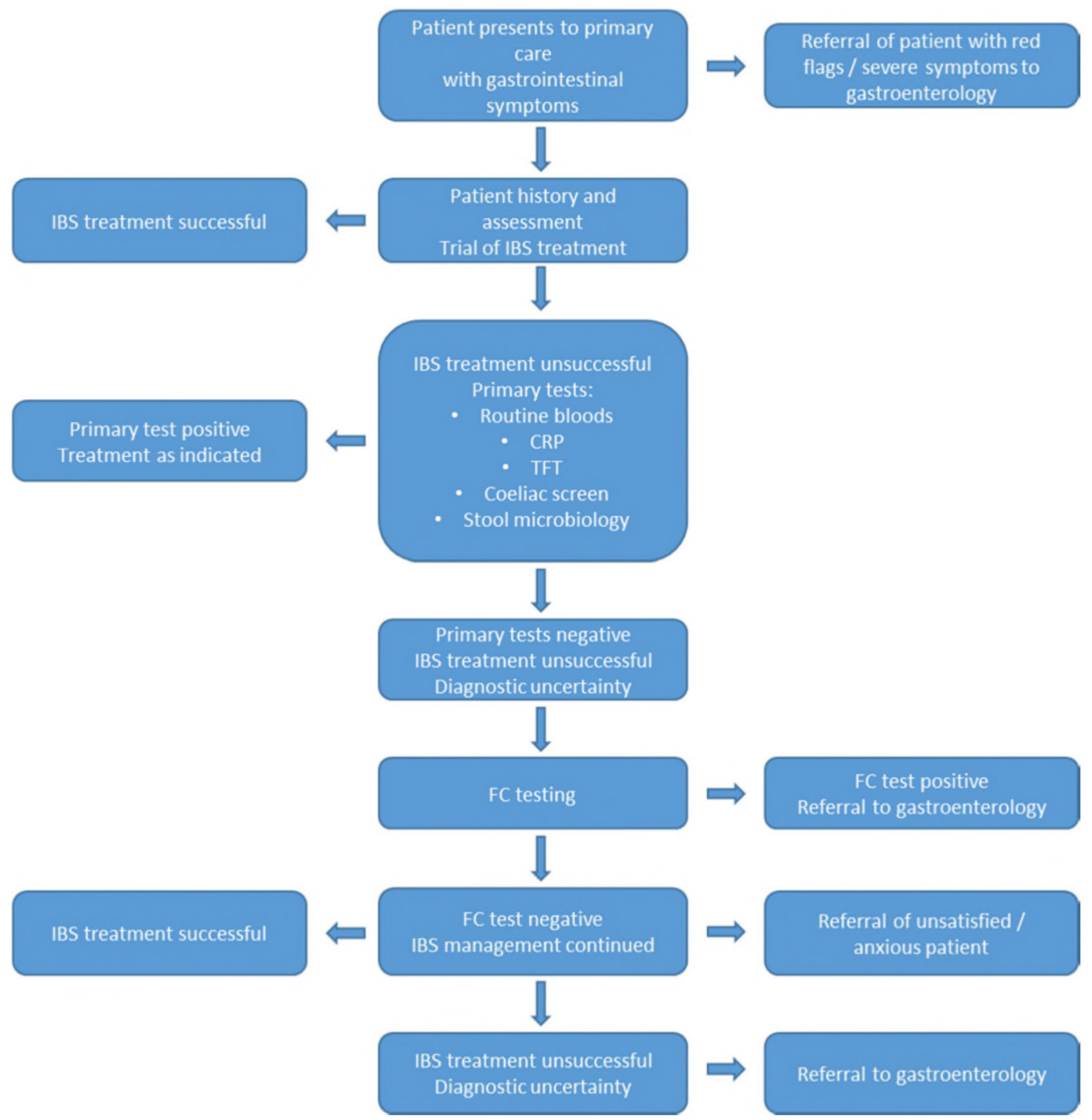

Figure 1 Indicative pathway of faecal calprotectin testing in primary care based on NICE guidelines (DG11) and expert opinion for adult patients presenting with chronic abdominal pain to primary care. CRP, C reactive protein; FC, faecal calprotectin; IBS, irritable bowel syndrome; NICE, National Institute for Health and Care Excellence; TFT, thyroid function test. 
included by Waugh et $a l^{18}$ for inclusion and searched MEDLINE, EMBASE, Cochrane Library and Web of Science from 1 September 2012 to 31 May 2017 (online supplementary 1). Eligible studies from auto-alerts were included up until 31 January 2018. Reference lists of included studies were checked.

Studies were assessed for eligibility independently and in duplicate, and any disagreements were resolved by consensus. Records rejected at full-text stage and reasons for exclusion were documented.

We included studies which measured FC levels in stool samples to detect IBD in adult patients (with $\geq 80 \%$ of study population 18-60 years) with chronic (at least 6-8 weeks) abdominal symptoms not yet diagnosed in primary or secondary care. The reference standard to verify $\mathrm{FC}$ test outcomes was colonoscopy with histology, other imaging technologies and follow-up. Our main outcomes of interest were sensitivity and specificity.

Data were extracted by $\mathrm{KF}$ on prespecified data extraction sheets (online supplementary 2) which were checked by HF. For studies reporting sensitivity, specificity, positive and negative predictive values and a total number of included patients, $2 \times 2$ tables of true positives, false positives (FPs), false negatives and true negatives were calculated. For studies reporting test accuracy at a lower and an upper cut-off, $2 \times 2$ data tables were extracted for the lower and the upper cut-off. Authors of relevant studies with missing $2 \times 2$ data were contacted to request data.

Quality was assessed independently and in duplicate by two reviewers using the Quality Assessment of Diagnostic Accuracy Studies-2 criteria, ${ }^{19}$ which included definitions for the signalling questions to match the review question.

\section{Patient involvement}

A patient advisory group was actively involved in the funding application for the project of which this study is a part. Two representatives of the patient advisory group will be involved in dissemination of findings at local and national events for patients with IBD.

\section{Analysis}

Studies were considered under three main clinical questions with different definitions of the target and non-target conditions: FC testing to differentiate: (1) IBD from IBS, (2) IBD from non-IBD conditions and (3) organic from non-organic conditions. Different FC test assays were handled independently.

We used Review Manager V.5.3 (The Cochrane Collaboration, The Nordic Cochrane Centre, Copenhagen, Denmark) to produce paired forest plots of sensitivity and specificity. All analyses were performed in R V.3.4.1 (Vienna, Austria). ${ }^{20}$ Meta-analyses were undertaken using a bivariate random effects model ${ }^{21}$ using the package glmer ${ }^{22}$ with a minimum of five studies required for meta-analysis. Different assays and clinical questions were considered separately. No overall summary estimates of sensitivity and specificity were produced due to heterogeneity. Test accuracy was explored at the commonly used thresholds of 50 and $100 \mu \mathrm{g}$ FC per $\mathrm{g}$ stool sample $(\mu \mathrm{g} / \mathrm{g})$. Outputs from meta-analyses were entered into Review Manager to produce receiver operating characteristic (ROC) plots.

Heterogeneity was explored by meta-regression analyses with assay type and clinical question added as covariates in turn. We tested the assumption of equal variances (online supplementary 3) suggesting this was reasonable for the data set. Additional models assuming unequal variances did not converge.

Each meta-analysis considered only one outcome per study. However, a number of studies reported $2 \times 2$ data for multiple test assays and clinical questions. We, therefore, undertook an exploratory sensitivity analysis. First, we produced a list of all possible combinations of test assay and clinical question for each included study at a common threshold (some studies reported outcomes for one test assay and clinical question contributing one possible combination while others reported results for up to three test assays and two clinical questions resulting in 6 possible combinations). We then ran a random sample of 25000 meta-analyses out of a possible 10 million picking one outcome per study at random in each round of meta-analysis and considering all combinations of test assays and clinical questions reported in the studies. Pairs of sensitivity and specificity from these meta-analyses were plotted in an ROC plot and a two-dimension density plot to take account of the different definitions of disease and test assays and to allow visualisation of the variability in the data.

\section{RESULTS}

Figure 2 provides the Preferred Reporting Items for Systematic Reviews and Meta-Analyses ${ }^{23}$ flow diagram of study selection. See online supplementary 4 for a list of records rejected at full-text stage and reasons for exclusion. Out of 2168 unique records, 38 studies were eligible for inclusion. The summary table 1 reveals extensive heterogeneity in all major aspects of test accuracy studies. Study characteristics by study can be found in online supplementary 5 .

Figure 3 summarises the assessment of risk of bias and applicability (online supplementary 6 for the assessment by study). Overall, the risk of bias was high or uncertain across all four domains in at least $50 \%$ of studies. Concerns about the applicability of the patient population to a primary care setting were high in about $75 \%$ of studies.

The forest plots in online supplementary 7 describe the complete evidence on test accuracy from the 38 included studies reporting all the thresholds, assays and clinical questions explored. Overall, as might be expected, sensitivity decreases and specificity increases as the threshold increases. FC testing appears to be more accurate in the detection of the more precise clinical construct of IBD than in the detection of organic disease as a whole.

Two studies ${ }^{24}$ compared five and six different test assays at a common threshold of $50 \mu \mathrm{g} / \mathrm{g}$. This allows for a test comparison in the same study population under 

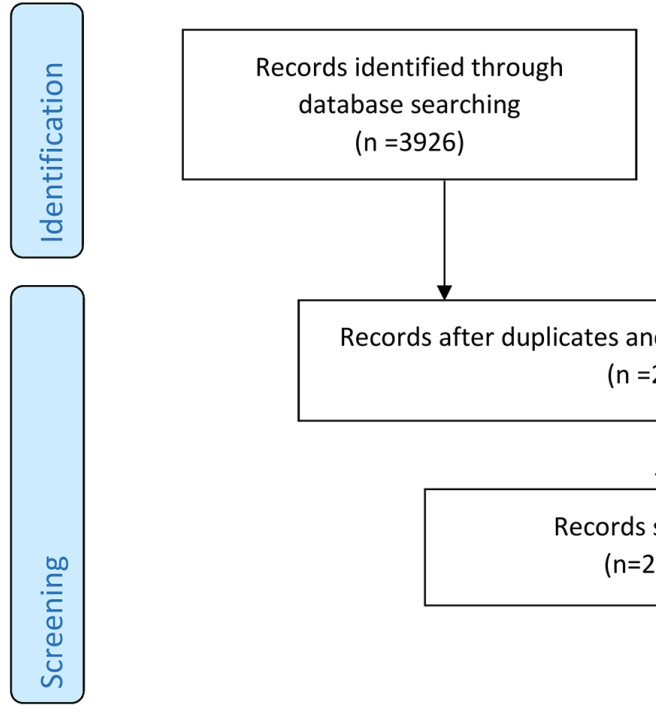

Additional records identified through auto alerts $(n=4)$
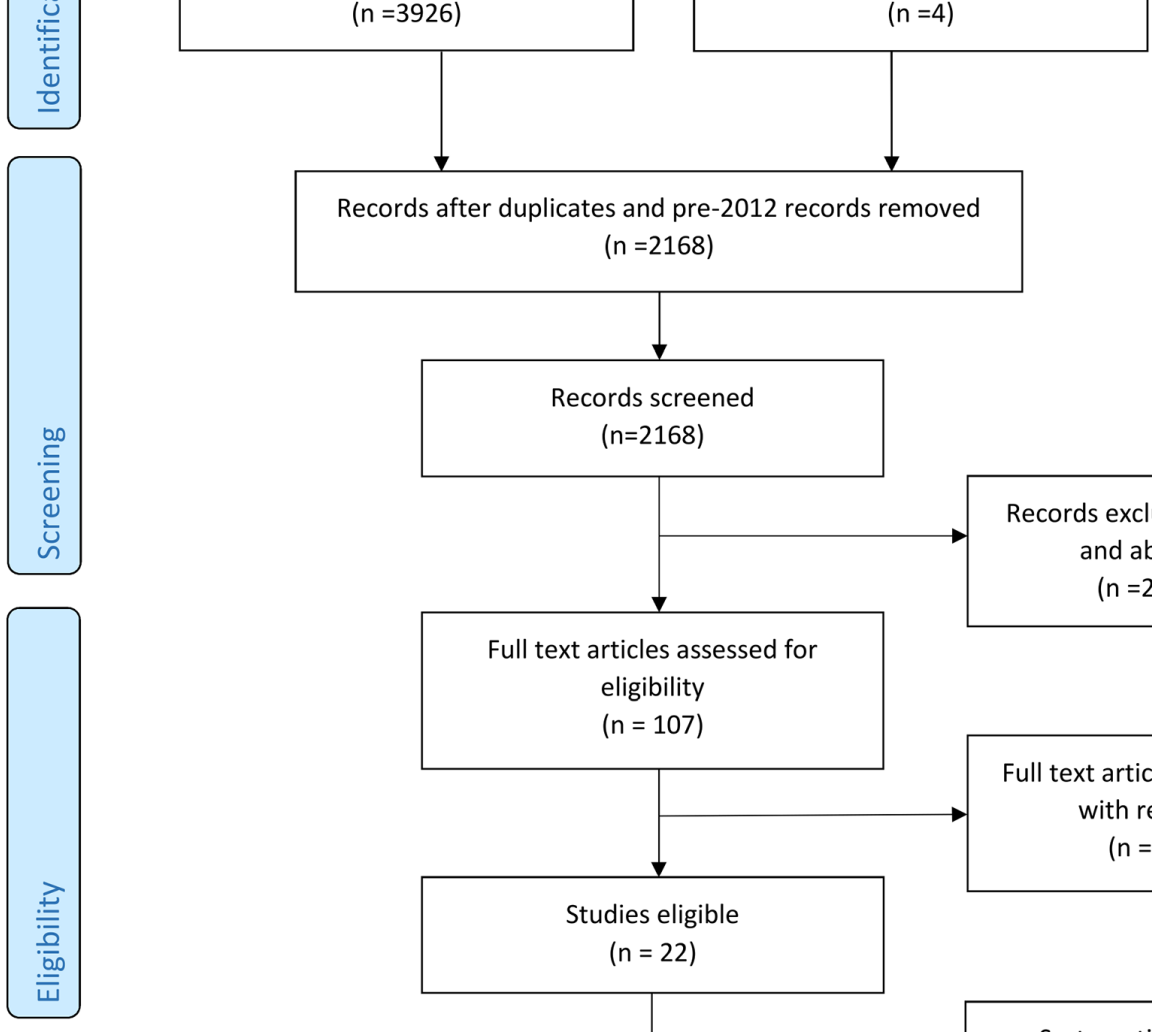
Records after duplicates and pre-2012 records removed
\[ (\mathrm{n}=2168) \]
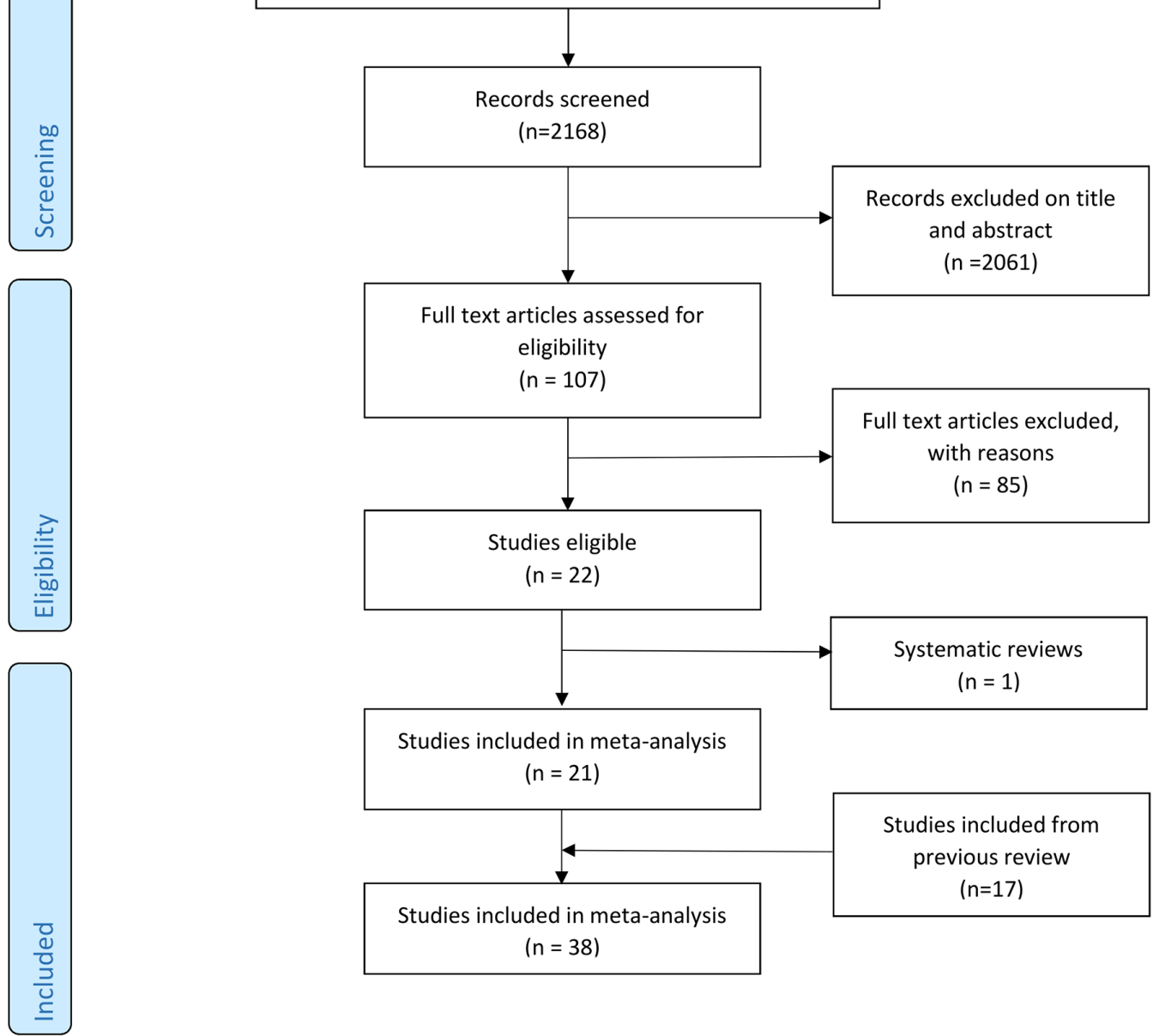

Full text articles assessed for eligibility $(n=107)$
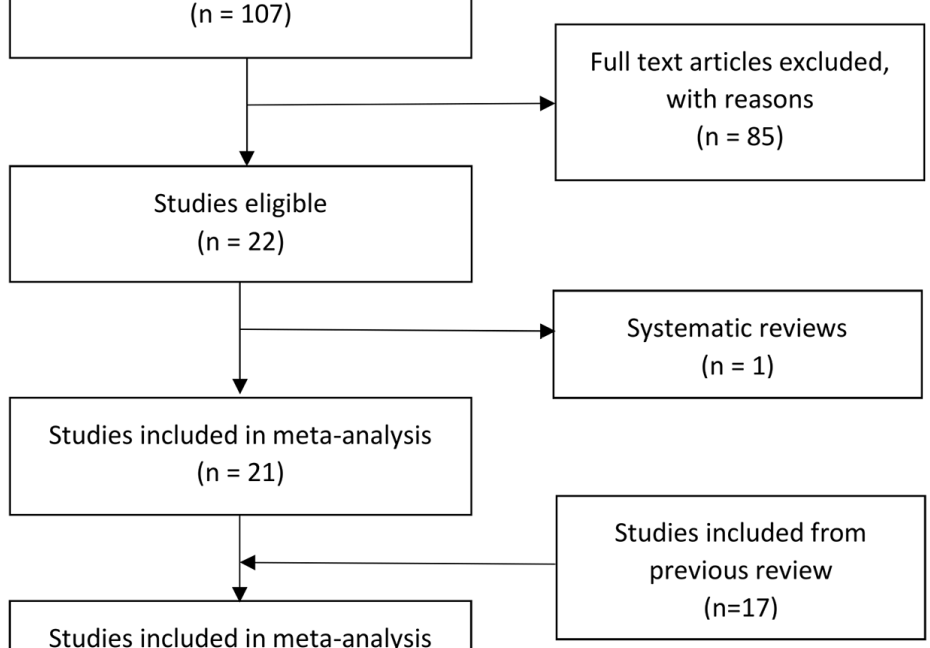

Studies included in meta-analysis $(n=38)$

Figure 2 Flow diagram of study inclusion.

similar study conditions. It appears that the specificity varies to a greater extent than the sensitivity (figure 4). Both studies reported medium to strong correlations but low agreement between assays, meaning that analysing the same sample with different FC test assays will result in different values for FC.

\section{Meta-analysis at $\mathbf{5 0} \mu \mathrm{g} / \mathrm{g}$ threshold}

Out of 38, 28 studies reported test accuracy at the common $50 \mu \mathrm{g} / \mathrm{g}$ cut-off and could be considered for meta-analysis. Meta-analyses were undertaken separately for test assays and for clinical questions to explore heterogeneity and to allow for multiple outcomes from a number of studies.

All 18 assays were considered in the meta-analysis investigating effect of assay type on summary estimates of test accuracy. (This was based on the assumption that the clinical question is generic and one outcome was picked per study for analysis). Four assays (PhiCal, EK-CAL, Quantum-Blue and EliA) with five or more studies each could be considered in the comparison (online supplementary 8 for $2 \times 2$ data). Nineteen studies contributed data. ${ }^{1024-41}$ Five studies ${ }^{1025313940}$ contributed data to two different assays. Figure 5 depicts the pairs of sensitivity and FP rates of contributing studies in the ROC space, with summary estimates by assay type. At the global $50 \mu \mathrm{g} / \mathrm{g}$ threshold, test performance appeared to vary slightly across assays (table 2). Quantum-Blue had the highest summary estimate for sensitivity with 0.94 (95\% CI 0.75 to 0.99$)$ but also the lowest specificity $(0.67,95 \%$ CI 0.56 to 0.76$)$. The greatest difference in sensitivity $(9 \%)$ was between EK-CAL ELISA and Quantum-Blue point-of-care test (POCT) while the greatest difference in specificity (21\%) was between PhiCal ELISA and Quantum-Blue 
Table 1 Summary of study characteristics of included studies addressing the test accuracy question of FC testing in the detection of IBD

\begin{tabular}{ll|}
\hline Study characteristic & Outcome \\
\hline Study characteristics: & \\
\hline Publication type (studies) & 32 \\
$\quad$ Full text & 6 \\
\hline Abstract & $2000-2018$ \\
\hline Year of publication (range) & $31-1031$ \\
\hline Population size (range) & \\
Geographical region (studies): & 14 \\
\hline UK & 16 \\
\hline Rest of Europe & 2 \\
Asia & 2 \\
Middle East & 1 \\
North Africa & 1 \\
\hline Russia & 1 \\
\hline Canada & 1 \\
\hline USA &
\end{tabular}

Patient characteristics:

\begin{tabular}{|c|c|}
\hline Age (range) & 14-97 years \\
\hline IBD prevalence (range) & $2.1 \%-76 \%$ \\
\hline \multicolumn{2}{|l|}{ FC assay type (studies)*: } \\
\hline \multicolumn{2}{|l|}{ Immunoassays } \\
\hline ELISA & 32 \\
\hline FEIA & 5 \\
\hline CLIA & 3 \\
\hline PETIA & 2 \\
\hline РОCT & 10 \\
\hline \multicolumn{2}{|l|}{ Setting (studies) $\dagger$} \\
\hline Primary care & 5 \\
\hline Secondary care & 29 \\
\hline Outpatients and inpatients & 12 \\
\hline Referred patients & 17 \\
\hline Mix & 2 \\
\hline
\end{tabular}

Target condition (studies)*:

\begin{tabular}{lr} 
IBD & 23 \\
Inflammatory disease & 5 \\
Organic disease & 19 \\
\hline
\end{tabular}

Non-target condition (studies)*

\begin{tabular}{lr}
\hline IBS & 12 \\
\hline Functional disease & 2 \\
\hline Non-IBD & 12 \\
\hline Non-organic disease & 16 \\
\hline Non-inflammatory disease & 5 \\
\hline Other & 3 \\
\hline FC test data collection (studies): & 1 \\
$\begin{array}{l}\text { Prospectively for patients with eligible } \\
\text { symptoms in primary care }\end{array}$ \\
\hline
\end{tabular}

Continued
Table 1 Continued

\begin{tabular}{ll}
\hline Study characteristic & Outcome \\
\hline $\begin{array}{l}\text { Prospectively for patients at time of referral in } \\
\text { primary care }\end{array}$ & 1 \\
$\begin{array}{l}\text { Retrospectively of routine FC tests in primary } \\
\text { or secondary care }\end{array}$ & 10 \\
$\begin{array}{l}\text { Prospectively prior to a planned colonoscopy } \\
\text { in secondary care }\end{array}$ & 23 \\
$\begin{array}{l}\text { Prospectively during the assessment for the } \\
\text { need of colonoscopy in secondary care }\end{array}$ & 1 \\
Unclear & 2 \\
Reference standard (studies): & 13 \\
Colonoscopy with biopsy & 7 \\
Colonoscopy \pm biopsy & 8 \\
Endoscopy+otherimaging tests & 3 \\
Endoscopy+follow-up & 5 \\
Endoscopy+otherimaging tests+follow-up & 2 \\
\hline Unclear
\end{tabular}

*Some studies evaluated multiple tests/clinical questions. †Two studies were unclear about the setting.

CLIA, chemiluminescent immunoassay; FC, faecal calprotectin; FEIA, fluorescence enzyme immunoassay; IBD, inflammatory bowel disease; IBS, irritable bowel syndrome; PETIA, particleenhanced turbidimetric immunoassay; POCT, point-of-care test.

POCT suggesting greater variation in specificity than in sensitivity across assays. Whether these differences are due to differing performance of test assays or due to methodological issues in study design is difficult to ascertain. The differences are large enough to suggest that test assays should not be treated as equivalent.

All reported $2 \times 2$ data (online supplementary 9 ) for the clinical questions IBD versus IBS, IBD versus non-IBD and organic versus non-organic disease were considered in the meta-analysis investigating the effects of the different clinical questions on summary estimates of test accuracy under the assumption that the test is generic. Twenty-eight studies were included at the common threshold of $50 \mu \mathrm{g} / \mathrm{g} .{ }^{1024-50}$ A total of 10 studies $^{30} 323436374345-48$ contributed information to more than one category. The ROC plot in figure 6 summarises the summary estimates for the comparison of the three clinical questions and the pairs of sensitivity and FP rates for the contributing studies. Table 2 shows that specificity for IBD versus non-IBD $(0.67,95 \%$ CI 0.58 to 0.75$)$ is lower than for IBD versus IBS $(0.76,95 \%$ CI 0.66 to 0.84$)$, acknowledging that CIs overlap, suggesting that broadening the definition of the non-target condition may produce more FPs as other non-IBD intestinal conditions are included. On the other hand, sensitivity for organic disease $(0.80$, $95 \%$ CI 0.76 to 0.84$)$ is lower than for IBD $(0.97,95 \%$ CI 0.91 to 0.99 ) suggesting that some organic disease will be missed with FC testing as organic conditions, including adenomas and diverticulosis, are not typically associated 


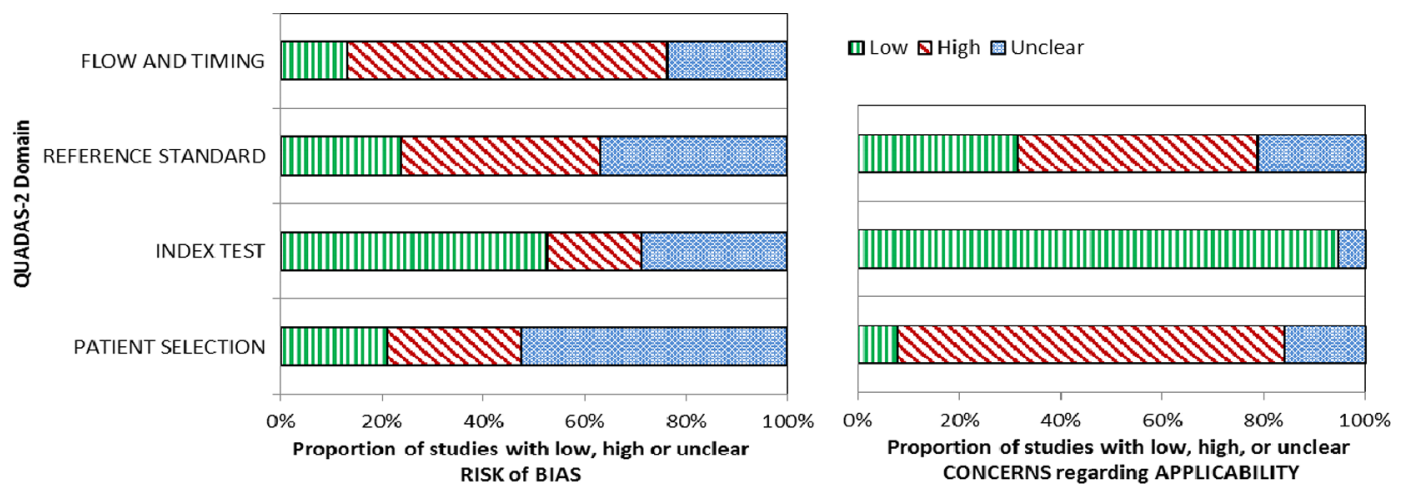

Figure 3 Overview of risk of bias and applicability concern of included studies according to QUADAS-2. ${ }^{19}$ QUADAS-2, Quality Assessment of Diagnostic Accuracy Studies-2.

with inflammation. Considering different definitions of the clinical questions results in greater variation in sensitivity than specificity meaning that widening the target condition had a greater impact on test accuracy than changing the non-target condition (IBS vs non-IBD vs non-organic disease).

\section{Meta-analysis at $100 \mu \mathrm{g} / \mathrm{g}$ threshold}

Eleven studies ${ }^{30} 32354345-4851-53$ reported test accuracy at a threshold of $100 \mu \mathrm{g} / \mathrm{g}$ (online supplementary 10 for $2 \times 2$ data). Of these, five contributed to more than one category of clinical questions. As expected raising the threshold to $100 \mu \mathrm{g} / \mathrm{g}$ reduces sensitivity and increases specificity for all clinical questions (table 2). Insufficient numbers of studies at the $100 \mu \mathrm{g} / \mathrm{g}$ were available to explore assay type.

\section{Meta-regression and exploratory sensitivity analysis}

Results from separate meta-analyses and from meta-regression analyses with equal variances showed similar results (table 2 and online supplementary 3 ).

Ten studies reported results for multiple clinical questions and eight studies compared multiple tests. Our approach of randomly selecting one test or one clinical question per study for meta-analysis uses only one set of evidence per study. Choosing a different set might have resulted in different outcomes and conclusions as the meta-analyses suggest that it might not be appropriate to consider tests and questions alike. In an attempt to capture this variation, figure 7 displays results of 25000 meta-analyses of 28 studies (online supplementary 11 for $2 \times 2$ data) picking one outcome per study at random for each round of meta-analysis. The results show overall high sensitivity and specificity irrespective of test assay and clinical question (Panel A) with slightly greater variation in sensitivity than specificity (Panel B). Median sensitivity and specificity of the 25000 analyses and ranges were 0.9 (min 0.85, $\max 0.94)$ and $0.76(\min 0.73, \max 0.79)$, respectively.

\section{Primary versus secondary care}

Five of the eligible studies were from primary care settings. ${ }^{3544474853}$ The studies were too heterogeneous for meta-analysis. In an attempt to compare test performance of FC testing in primary care with secondary

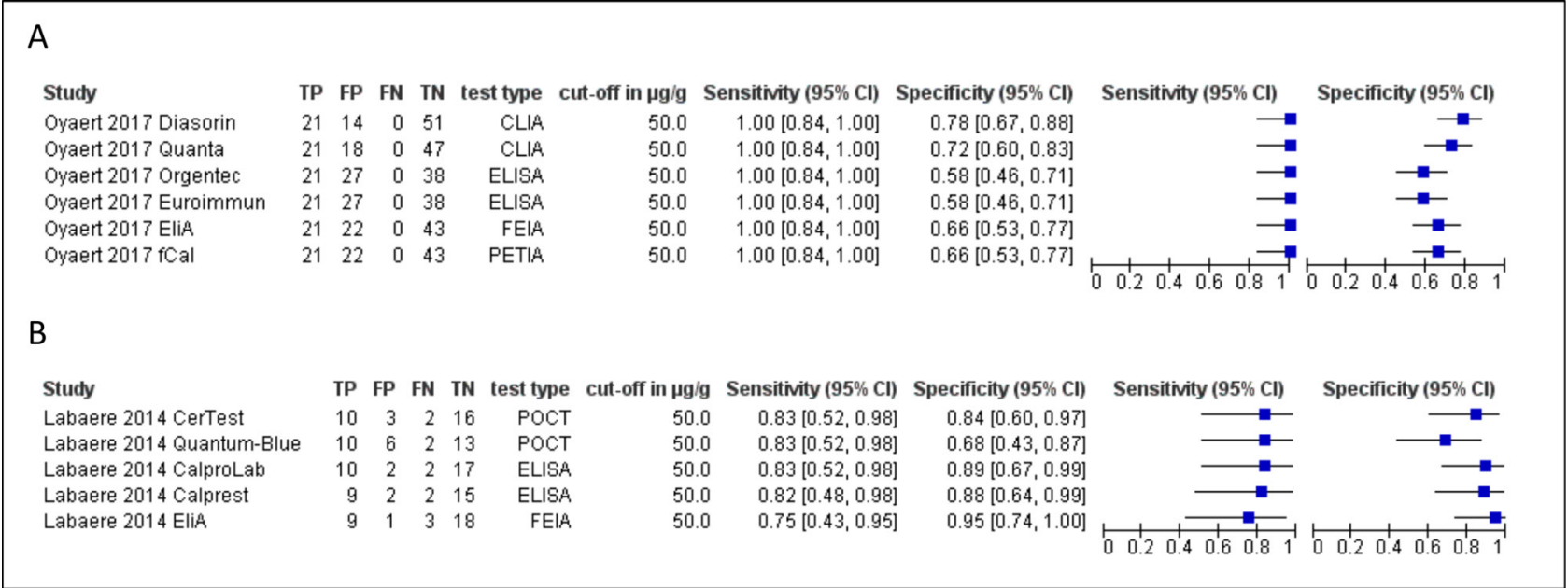

Figure 4 Forest plot of (A) Oyaert et al ${ }^{24}$ comparing six FC tests and (B) Labaere et al ${ }^{25}$ comparing five FC tests at cut-off $50 \mu \mathrm{g} / \mathrm{g}$. CLIA, chemiluminescent immunoassay; FC, faecal calprotectin; FEIA, fluorescence enzyme immunoassay; FN, false negative; FP, false positive; PETIA, particle-enhanced turbidimetric immunoassay; POCT, point-of-care test; TP, true positive; TN, true negative. 


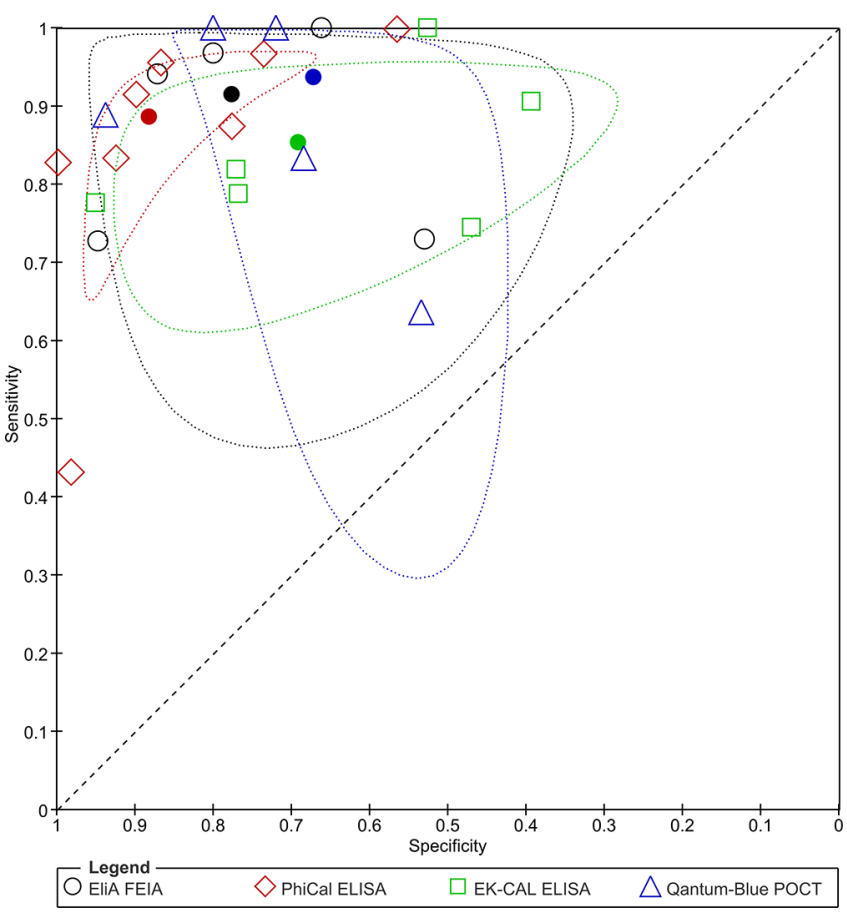

Figure 5 Receiver operating characteristic plot of sensitivity and specificity by test (filled shapes present summary estimates with $95 \%$ confidence region). FEIA, fluorescence enzyme immunoassay; POCT, point-of-care test.

care only one study qualified. ${ }^{47}$ The study evaluated the routine use of FC testing in primary care and reported lower sensitivity of 0.72 but comparable specificity of 0.65 for differentiating IBD from non-IBD at a threshold of $50 \mu \mathrm{g} / \mathrm{g}$ when compared with our pooled estimates across different test assays including all settings (sensitivity 0.95 , $95 \%$ CI 0.88 to 0.98 and specificity $0.67,95 \%$ CI 0.58 to $0.75)$. However, indication for testing and the place of the FC test in the patient pathway were unclear in this study and the study may suffer from differential verification bias due to concerns over the reference standard. The authors explained the low sensitivity with the fact that general practitioners may have referred patients with high suspicion of IBD without testing. Another reasonable explanation could be the greater number of apparently milder IBD cases in primary care in which FC testing is falsely negative, reducing sensitivity in this setting and demonstrating the impact of the different spectrum of disease on test accuracy estimates. ${ }^{54}$

\section{DISCUSSION}

None of the 38 included studies sufficiently addressed the review question of test accuracy of FC testing in a primary care pathway. The studies recruited patients with a different spectrum of disease from that anticipated for primary care, focused on disease groups which were broader than the intended IBD group or did not verify all patients with the preferred reference standard. Furthermore, the evidence was highly heterogeneous. Studies varied in thresholds and assays used, as well as in target conditions and settings. Eighteen different test assays were studied, although similar they could not be considered generic for meta-analysis. Clinical questions were analysed as three distinct categories which were related but which could not be pooled for meta-analysis. A number of studies compared multiple tests and/ or explored different clinical questions. Picking one test and one clinical question per study for meta-analysis was unjustifiable. We favoured an innovative approach which allowed us to explore the whole breadth of evidence and showed that irrespective of clinical question and test assay, test accuracy of FC testing was high. However, the variation in specificity could translate into considerable uncertainty of FPs when scaled up to population testing, while the variation in sensitivity reflects the limited value of FC testing for other organic conditions.

The review has a number of limitations. Due to heterogeneity, we decided not to pool across tests and clinical questions. Instead, we conducted exploratory analyses which demonstrated that the test assay and clinical question may affect the summary sensitivity and specificity by as much as $9 \%$. We were also unable to compare primary and secondary care studies due to the small number of heterogeneous studies in primary care. The meta-regression analyses assumed equal variances between categories of tests and clinical questions and this was supported by a statistical comparison. However, the power of this test is dependent on the number of studies per subgroup and it is possible that this assumption could be challenged with a larger data set. Finally, the categorisation into different clinical questions was subjective because the disease categories are ill defined and studies' definitions of conditions and groups of conditions varied.

This review is broadly in line with the approaches and interpretation of the evidence of the previous review. ${ }^{18}$ However, Waugh et al reported pooled estimates of sensitivity $(0.93,95 \% \mathrm{CI} 0.83$ to 0.97$)$ and specificity $(0.94$, $95 \%$ CI 0.73 to 0.99 ) across tests for the differential diagnosis of IBD versus IBS for five secondary care studies and this was the basis for national decisions to introduce FC testing in primary care. When compared with our equivalent analysis of 11 studies, we found comparable sensitivity $(0.97,95 \%$ CI 0.91 to 0.99$)$ but considerably lower specificity $(0.76,95 \%$ CI 0.66 to 0.84$)$ suggesting that FPs might be more of a concern than previously concluded. Thus, the predicted reduction in colonoscopies and subsequent cost savings may not be realised as a result of introducing FC testing into the primary care pathway in the UK. ${ }^{18}$ Furthermore, this review found enough disagreement between tests to caution against treating tests as equivalent. Issues in homogenisation, dilutions and extraction prior to analysis as well as lack of standardisation of different assays contribute to these differences. Recommended cut-off values would have to be determined locally until these issues are resolved. ${ }^{24}$

None of the primary care studies assessed FC testing for the differential diagnosis of IBD versus IBS. The comparison of IBD versus non-IBD might reflect the 


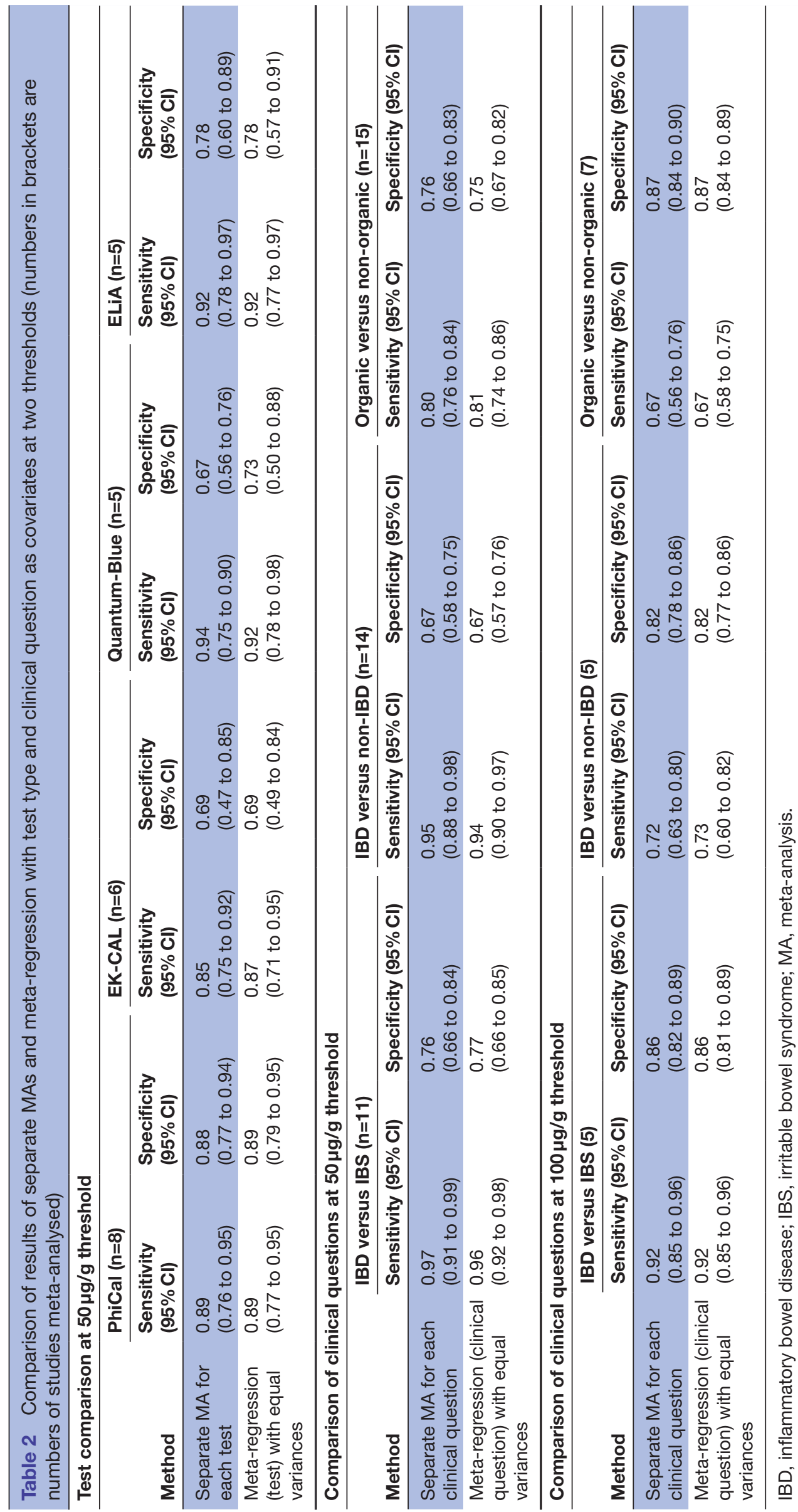

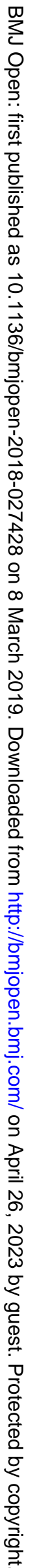




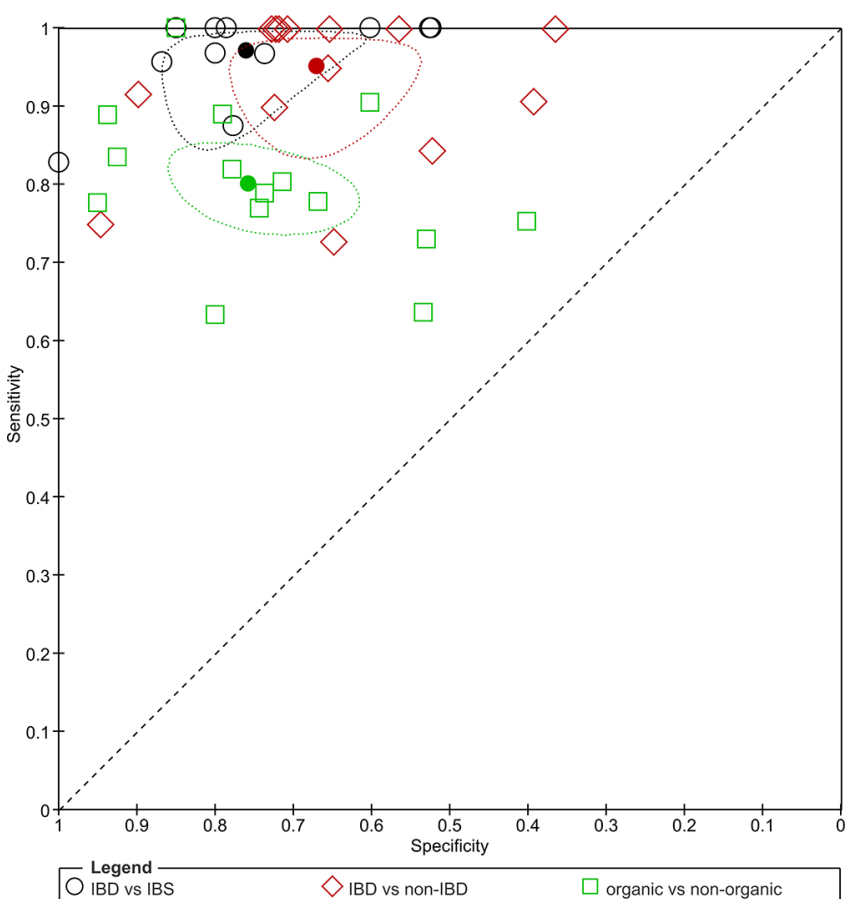

Figure 6 Receiver operating characteristic plot of sensitivity and specificity by clinical question (filled shapes present summary estimates with $95 \%$ confidence region). IBD, inflammatory bowel disease; IBS, irritable bowel syndrome.

clinical situation for FC testing more accurately, but produces more FPs as FC levels might be raised in diverticular disease, coeliac disease, rectal adenocarcinoma, non-specific inflammation, and others. ${ }^{35} 53$ The meaning of the additional FPs due to non-IBD inflammatory conditions is debatable in the context of clinical practice where incidental findings of true disease would not be classed as an FP test outcome but would prompt further investigations.

NICE recently endorsed a cut-off of $100 \mu \mathrm{g} / \mathrm{g}$ for use in primary care in England and Wales, based on a primary care study which showed a $43 \%$ reduction in FC test positives compared with a threshold of $50 \mu \mathrm{g} / \mathrm{g} .{ }^{47}$ We investigated test accuracy at this cut-off including all settings and demonstrated an increase in specificity by at least $10 \%$, while the magnitude of the resulting decrease in sensitivity was more uncertain.

Referral of FC tested patients was consistent in three UK primary care studies $\left(41 \%,{ }^{53} 42 \%{ }^{35}\right.$ and $48 \%{ }^{47}$ with over $25 \%$ of FC negative patients referred. This raises concerns about the impact of FC testing on colonoscopy rates as a considerable proportion of referred patients with negative FC levels were further investigated. Inappropriate use of FC testing as a screening test rather than to rule out IBD has been proposed as an explanation. ${ }^{55}$ However, the number of investigations of referred FC negatives varied greatly among the three studies from $19 \%{ }^{47}$ to $46 \%{ }^{35}$ and even $71 \%{ }^{53}$ suggesting perhaps unsurprisingly, that the number of potentially unnecessary colonoscopies may be at least as dependent on secondary care decision-making as on the availability of FC testing in primary care.

The applicability of the spectrum of secondary care patients to a primary care setting is questionable even if studies investigated referred patients. First, general practitioners might have tested more patients than they referred and, second, patients referred came from different pathways including cancer pathways. Consequently, the patient population of the included studies resembled a continuum of primary to secondary care patients of which only some might plausibly resemble the primary care spectrum. Additionally, primary care populations may be different in different countries, it would,
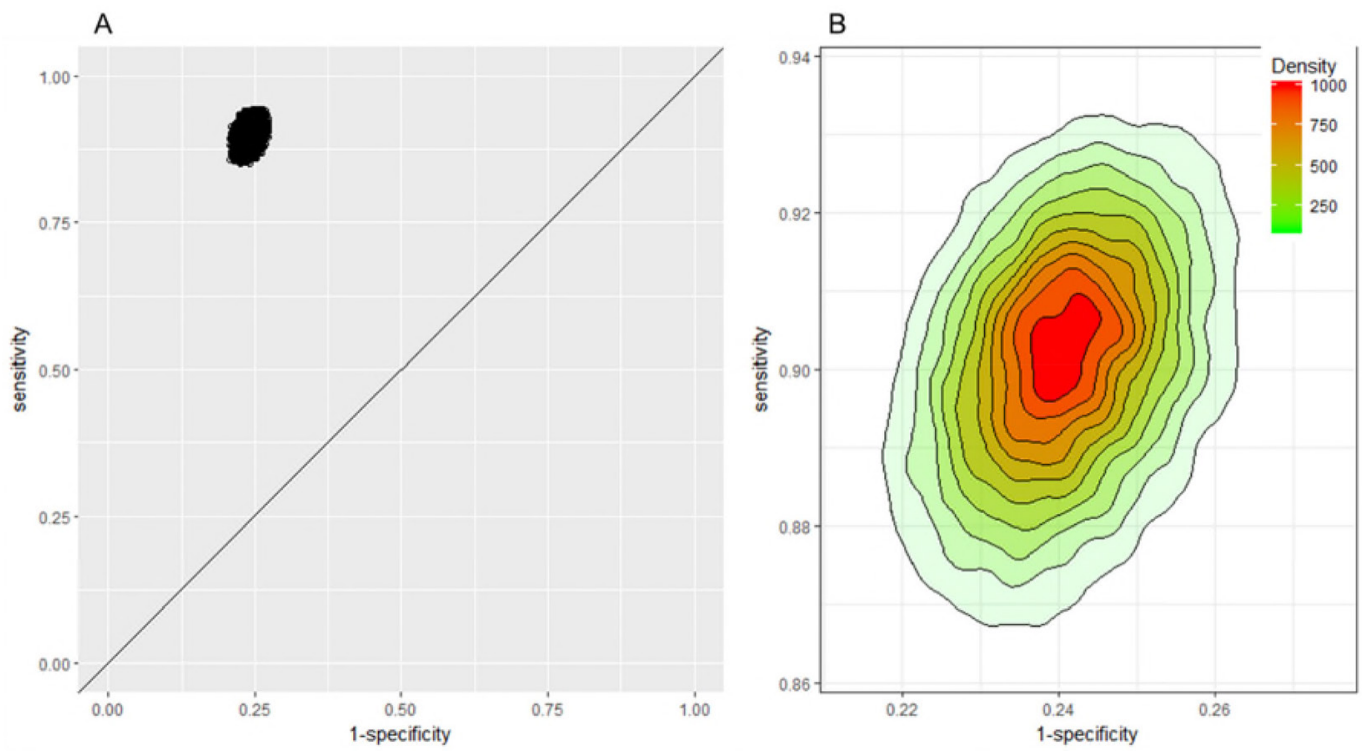

Figure 7 Pairs of sensitivity and specificity of 25000 meta-analyses of 28 studies picking one outcome per study at random for each round of meta-analysis at a threshold of $50 \mu \mathrm{g} / \mathrm{g}$. (A) Receiver operating characteristic plot, (B) 2D density plot scaled to illustrate spread and density (where the highest density represents a probability of 1000/25 000). 2D, two dimensions. 
therefore, be useful to identify those studies which are relevant to primary care irrespective of the actual study setting, for instance, using tailored meta-analysis. ${ }^{56} 57$ Furthermore, the statistical validity of any summary estimates could be evaluated using a recently developed cross-validation technique ${ }^{58}$ Only five studies recruited subjects from primary care populations and analysed the general practitioners' decisions on whether to test and whether to refer. ${ }^{3544} 474853$ We found lower sensitivity for detection of disease in these studies compared with meta-analyses of all studies.

Despite the publication of primary care studies since the Waugh et al review, ${ }^{18}$ we are still lacking evidence on the defined role of FC testing in the primary care pathway for the detection of IBD. This is a concern given that it is prescribed by local and national guidance in the UK and the roll-out of a national algorithm endorsed by NICE, the National Health Service England Chief Scientific Officer and the British Society of Gastroenterologists is imminent to improve spread and adoption of FC testing nationally. ${ }^{59}$ The decision to introduce FC testing in primary care might have been based on overoptimistic assessments of potential test accuracy in this setting. Evidence for the test accuracy of FC testing in primary care which considers the revised cut-off of $100 \mu \mathrm{g} / \mathrm{g}$ is needed before any recommendations for use in primary care can be made. Potentially, test accuracy studies in primary care are hampered because verification of FC test outcomes with colonoscopy may not be feasible in all tested patients. Therefore, other reference standards for assessing the performance of $\mathrm{FC}$ testing in primary care are also needed.

Acknowledgements We would like to thank the patient advisory group of the project 'What is the role of faecal calprotectin testing in primary care' for their input.

Contributors $\mathrm{KF}$ and $\mathrm{HF}$ undertook the review. KF and $\mathrm{BHW}$ carried out the analysis. KF, BHW, ST-P and AC contributed to the interpretation of the findings. KF drafted the manuscript. All authors critically revised the manuscript and approved the final version. KF takes responsibility for the integrity and accuracy of the data analysis. KF acts as guarantor. The corresponding author attests that all listed authors meet authorship criteria and that no others meeting the criteria have been omitted.

Funding This study was funded by the NIHR (NIHR Doctoral Research Fellowship, KF, DRF-2016- 09-038).

Disclaimer The views expressed are those of the author(s) and not necessarily those of the NHS, the NIHR or the Department of Health and Social Care. The funder had no role in the study design, data collection, data analysis and interpretation, writing of the report or the decision to submit of publication.

Competing interests $\mathrm{KF}$ is funded by the NIHR through a doctoral research fellowship. ST-P and AC are supported by the NIHR CLAHRC West Midlands initiative. ST-P is funded by the NIHR through a career development fellowship. BHW is funded by an MRC Clinician Scientist Fellowship award (MR/N007999/1).

Patient consent for publication Not required.

Provenance and peer review Not commissioned; externally peer reviewed.

Data sharing statement All data relevant to the study are included in the article or uploaded as supplementary information.

Open access This is an open access article distributed in accordance with the Creative Commons Attribution 4.0 Unported (CC BY 4.0) license, which permits others to copy, redistribute, remix, transform and build upon this work for any purpose, provided the original work is properly cited, a link to the licence is given, and indication of whether changes were made. See: https://creativecommons.org/ licenses/by/4.0/.

\section{REFERENCES}

1. Ungaro R, Mehandru S, Allen PB, et al. Ulcerative colitis. Lancet 2017;389:1756-70.

2. Torres J, Mehandru S, Colombel J-F, et al. Crohn's disease. The Lancet 2017;389:1741-55.

3. Frolkis AD, Dykeman J, Negrón ME, et al. Risk of surgery for inflammatory bowel diseases has decreased over time: a systematic review and meta-analysis of population-based studies. Gastroenterology 2013;145:996-1006.

4. Dahlhamer JM, Zammitti EP, Ward BW, et al. Prevalence of inflammatory bowel disease among adults aged $\geq 18$ years - United States, 2015. MMWR Morb Mortal Wkly Rep 2016:65:1166-9.

5. Burisch J, Jess T, Martinato M, et al. The burden of inflammatory bowel disease in Europe. J Crohns Colitis 2013;7:322-37.

6. Rubin GP, Hungin AP, Kelly PJ, et al. Inflammatory bowel disease: epidemiology and management in an English general practice population. Aliment Pharmacol Ther 2000;14:1553-9.

7. Molodecky NA, Soon IS, Rabi DM, et al. Increasing incidence and prevalence of the inflammatory bowel diseases with time, based on systematic review. Gastroenterology 2012:142:46-54. quiz e30.

8. van den Heuvel-Janssen HA, Borghouts JA, Muris JW, et al. Chronic non-specific abdominal complaints in general practice: a prospective study on management, patient health status and course of complaints. BMC Fam Pract 2006;7:12.

9. Janssen HA, Borghouts JA, Muris JW, et al. Health status and management of chronic non-specific abdominal complaints in general practice. Br J Gen Pract 2000;50:375-9.

10. Kok L, Elias SG, Witteman BJ, et al. Diagnostic accuracy of point-of-care fecal calprotectin and immunochemical occult blood tests for diagnosis of organic bowel disease in primary care: the Cost-Effectiveness of a Decision Rule for Abdominal Complaints in Primary Care (CEDAR) study. Clin Chem 2012;58:989-98.

11. Eskeland SL, Dalén E, Sponheim J, et al. European panel on the appropriateness of gastrointestinal endoscopy II guidelines help in selecting and prioritizing patients referred to colonoscopy--a quality control study. Scand J Gastroenterol 2014;49:492-500.

12. Konikoff MR, Denson LA. Role of fecal calprotectin as a biomarker of intestinal inflammation in inflammatory bowel disease. Inflamm Bowel Dis 2006;12:524-34.

13. Lichtenstein GR, Loftus EV, Isaacs KL, et al. ACG clinical guideline: management of Crohn's Disease in Adults. Am J Gastroenterol 2018;113:481-517.

14. The Gastroenterological Society of Australia. Australian guidelines for general practitioners and physicians: inflammatory bowel disease, forth edition. $2017 \mathrm{http} / / /$ cart.gesa.org.au/membes/files/Clinical\% 20Guidelines\%20and\%20Updates/2017_IBD_guidelines_DRAFT_ for_consultation_20102017.pdf (cited 08 Oct 2018).

15. Preiß J, Bokemeyer B, Buhr H, et al. Updated german clinical practice guideline on "Diagnosis and treatment of Crohn's disease". 2014 https://www.dgvs.de/wp-content/uploads/2016/11/DGVS Empfehlung_fuer_Diagnistik_und_Therapie_des_Morbus_Crohn.pdf (cited 08 Oct 2018).

16. Gomollón F, Dignass A, Annese V, et al. 3rd European Evidencebased Consensus on the Diagnosis and Management of Crohn's Disease 2016: Part 1: Diagnosis and Medical Management. J Crohns Colitis 2017:11:3-25.

17. National Institute for Health and Care Excellence (NICE). Faecal calprotectin diagnostic tests for inflammatory diseases of the bowel: NICE diagnostics guidance [DG11]. 2013 https://www.nice.org.uk/ guidance/dg11 (cited 17 Dec 2015)

18. Waugh N, Cummins E, Royle P, et al. Faecal calprotectin testing for differentiating amongst inflammatory and non-inflammatory bowel diseases: systematic review and economic evaluation. Health Technol Assess 2013;17:1-211.

19. Whiting PF, Rutjes AW, Westwood ME, et al. QUADAS-2: a revised tool for the quality assessment of diagnostic accuracy studies. Ann Intern Med 2011;155:529-36.

20. R Core Team. R: A language and environment for statistical computing. R Foundation for Statistical Computing, Vienna, Austria [computer program]. 2017 https://www.R-project.org/.

21. Chu H, Cole SR. Bivariate meta-analysis of sensitivity and specificity with sparse data: a generalized linear mixed model approach. $J$ Clin Epidemiol 2006;59:1331-2. author reply 2-3.

22. Partlett $C$, Takwoingi $Y$. Meta-analysis of test accuracy studies in $R$ : a summary of userwritten programs and step-by-step guide to using 
glmer. Version 1.0. 2016 http://methods.cochrane.org/sdt/ (cited 30 Jan 2018).

23. Mclnnes MDF, Moher D, Thombs BD, et al. Preferred Reporting Items for a Systematic Review and Meta-analysis of Diagnostic Test Accuracy Studies: The PRISMA-DTA Statement. JAMA 2018;319:388-96.

24. Oyaert M, Boel A, Jacobs J, et al. Analytical performance and diagnostic accuracy of six different faecal calprotectin assays in inflammatory bowel disease. Clin Chem Lab Med 2017;55:21:21.

25. Labaere D, Smismans A, Van Olmen A, et al. Comparison of six different calprotectin assays for the assessment of inflammatory bowel disease. United European Gastroenterol J 2014;2:30-7.

26. Turvill J. High negative predictive value of a normal faecal calprotectin in patients with symptomatic intestinal disease. Frontline Gastroenterol 2012;3:21-8.

27. Schoepfer AM, Trummler M, Seeholzer P, et al. Discriminating IBD from IBS: comparison of the test performance of fecal markers, blood leukocytes, CRP, and IBD antibodies. Inflamm Bowel Dis 2008;14:32-9.

28. Otten CM, Kok L, Witteman BJ, et al. Diagnostic performance of rapid tests for detection of fecal calprotectin and lactoferrin and their ability to discriminate inflammatory from irritable bowel syndrome. Clin Chem Lab Med 2008:46:1275-80.

29. Li XG, Lu YM, Gu F, et al. [Fecal calprotectin in differential diagnosis of irritable bowel syndrome]. Beijing Da Xue Xue Bao Yi Xue Ban 2006;38:310-3.

30. El-Badry A, Sedrak H, Rashed L. Faecal calprotectin in differentiating between functional and organic bowel diseases. Arab Journal of Gastroenterology 2010;11:70-3.

31. Burri E, Manz M, Rothen C, et al. Monoclonal antibody testing for fecal calprotectin is superior to polyclonal testing of fecal calprotectin and lactoferrin to identify organic intestinal disease in patients with abdominal discomfort. Clin Chim Acta 2013;416:41-7.

32. Kennedy NA, Clark A, Walkden A, et al. Clinical utility and diagnostic accuracy of faecal calprotectin for IBD at first presentation to gastroenterology services in adults aged 16-50 years. J Crohns Colitis 2015;9:41-9.

33. Dhaliwal A, Zeino Z, Tomkins C, et al. Utility of faecal calprotectin in inflammatory bowel disease (IBD): what cut-offs should we apply? Frontline Gastroenterol 2015;6:14-19.

34. Damms A, Bischoff SC. Validation and clinical significance of a new calprotectin rapid test for the diagnosis of gastrointestinal diseases. Int $J$ Colorectal Dis 2008;23:985-92.

35. Pavlidis P, Chedgy FJ, Tibble JA. Diagnostic accuracy and clinical application of faecal calprotectin in adult patients presenting with gastrointestinal symptoms in primary care. Scand J Gastroenterol 2013;48:1048-54.

36. Mowat C, Digby J, Strachan JA, et al. Faecal haemoglobin and faecal calprotectin as indicators of bowel disease in patients presenting to primary care with bowel symptoms. Gut 2016:65:1463-9.

37. De Sloovere MMW, De Smet D, Baert FJ, et al. Analytical and diagnostic performance of two automated fecal calprotectin immunoassays for detection of inflammatory bowel disease. Clin Chem Lab Med 2017;55:11:11.

38. Livzan MA, Lyalukova EA, Nechaeva G, et al. Calprotectin, transferrin, hemoglobin) in complex diagnosis of diseases of intestines]. Eksperimental'Naia i Klinicheskaia Gastroenterologiia 2015;3:34-8.

39. Oyaert $\mathrm{M}$, Trouvé $\mathrm{C}$, Baert $\mathrm{F}$, et al. Comparison of two immunoassays for measurement of faecal calprotectin in detection of inflammatory bowel disease: (pre)-analytical and diagnostic performance characteristics. Clin Chem Lab Med 2014;52:391-7.

40. Jang HW, Kim HS, Park SJ, et al. Accuracy of three different fecal calprotectin tests in the diagnosis of inflammatory bowel disease. Intest Res 2016;14:305-13.
41. Sostres C, Lue A, Barra Pardos MV, et al. Combination of FOBT and fecal calprotectin may be useful for reducing unnecessary colonoscopies in symptomatic patients. United European Gastroenterology Journal 2017;5(Suppl 1):A779.

42. Alrubaiy L, Malik A, Rees I, et al. Usefulness of fecal calprotectin in clinical practice in a district general hospital. Inflamm Bowel Dis 2012;18:S53-S54.

43. Banerjee A, Srinivas M, Eyre R, et al. Faecal calprotectin for differentiating between irritable bowel syndrome and inflammatory bowel disease: a useful screen in daily gastroenterology practice. Frontline Gastroenterol 2015;6:20-6.

44. Boyd A, Peplow C, Dhaliwal A, et al. Primary care faecal calprotectin pathway - A three year analysis. Colorectal Disease 2016;18:27.

45. Carroccio A, lacono G, Cottone M, et al. Diagnostic accuracy of fecal calprotectin assay in distinguishing organic causes of chronic diarrhea from irritable bowel syndrome: a prospective study in adults and children. Clin Chem 2003;49(6 Pt 1):861-7.

46. Caviglia GP, Pantaleoni S, Touscoz GA, et al. Fecal calprotectin is an effective diagnostic tool that differentiates inflammatory from functional intestinal disorders. Scand J Gastroenterol 2014;49:1419-24.

47. Conroy S, Hale MF, Cross SS, et al. Unrestricted faecal calprotectin testing performs poorly in the diagnosis of inflammatory bowel disease in patients in primary care. J Clin Pathol 2018;71.

48. Högberg C, Karling P, Rutegård J, et al. Diagnosing colorectal cancer and inflammatory bowel disease in primary care: The usefulness of tests for faecal haemoglobin, faecal calprotectin, anaemia and iron deficiency. A prospective study. Scand J Gastroenterol 2017; 52:69-75

49. Tibble JA, Sigthorsson G, Foster R, et al. Use of surrogate markers of inflammation and Rome criteria to distinguish organic from nonorganic intestinal disease. Gastroenterology 2002;123:450-60.

50. Tan M, Subramaniam Y, Cheong C, et al. The role of faecal calprotectin in a primary care referral pathway in the UK. United European Gastroenterology Journal 2016;4(5 Suppl 1):A443.

51. Limburg PJ, Ahlquist DA, Sandborn WJ, et al. Fecal calprotectin levels predict colorectal inflammation among patients with chronic diarrhea referred for colonoscopy. Am J Gastroenterol 2000;95:2831-7.

52. Rosenfeld G, Greenup AJ, Round A, et al. FOCUS: Future of fecal calprotectin utility study in inflammatory bowel disease. World $\mathrm{J}$ Gastroenterol 2016;22:8211-8.

53. Turvill J, O'Connell S, Brooks A, et al. Evaluation of a faecal calprotectin care pathway for use in primary care. Prim Health Care Res Dev 2016;17:428-36.

54. Willis BH. Spectrum bias--why clinicians need to be cautious when applying diagnostic test studies. Fam Pract 2008;25:390-6.

55. $\mathrm{Ng} \mathrm{S}$, Box B, Khan K. Faecal calprotectin-a good test used badly. Colorectal Disease 2017;19(S4):64.

56. Willis BH, Hyde CJ. Estimating a test's accuracy using tailored metaanalysis-How setting-specific data may aid study selection. J Clin Epidemiol 2014;67:538-46.

57. Willis BH, Hyde CJ. What is the test's accuracy in my practice population? Tailored meta-analysis provides a plausible estimate. $J$ Clin Epidemiol 2015;68:847-54.

58. Willis BH, Riley RD. Measuring the statistical validity of summary meta-analysis and meta-regression results for use in clinical practice. Stat Med 2017;36:3283-301.

59. Commissioning PC. Faecal calprotectin in primary care as a decision diagnostic for inflammatory bowel disease and irritable bowel syndrome. $2018 \mathrm{https}$ ://www.pcc-cic.org.uk/article/updatedguidance-help-gps-easily-diagnose-serious-bowel-conditions (cited 09 Jul 2018). 